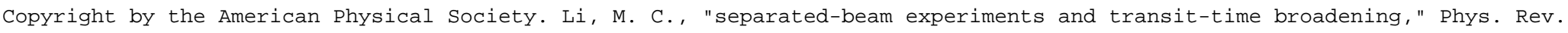
A 23, 2995 (1981); DOI: http://dx.doi.org/10.1103/PhysRevA.23.2995

PH Y S I CAL REVIE W A

\title{
Separated-beam experiments and transit-time broadening
}

\author{
Ming Chiang Li \\ Department of Physics, Virginia Polytechnic Institute and State University, Blacksburg, Virginia 24061
}

(Received 19. February 1980)

\begin{abstract}
Separated-beam experiments for both the Doppler-free two-photon and saturated absorptions are under consideration. In the Doppler-free two-photon absorption, the experimental arrangement is important. Such importance has not been recognized before. Three different arrangements are discussed here. In the first one, the separated-beam experiment is not coherent and the Ramsey fringe variation cannot be observed. In the second, the transit-time broadening is present and the Ramsey fringe variation depends on the atom's time of flight as well as the transit time. Finally, in the third arrangement, the transit-time broadening is eliminated and the Ramsey fringe variation depends on the atom's time of flight alone. These examples have shown that in the Doppler-free twophoton absorption, the elimination of the transit-time broadening is through a mechanism other than the beam separation. In the saturated absorption the situation is quite different. Even in the presence of transit-time broadening, the Ramsey fringe spacing depends only on the atom's time of flight.
\end{abstract}

\section{INTRODUCTION}

The atomic and molecular absorptions initiated by coherent laser beams have become a subject for active research. When an atom or molecule, interacts with coherent beams, it gives rise to a coherent phenomenon. ${ }^{1}$ The transition rate becomes dependent on an external phase which can be varied directly. The variation leads to a direct determination of the phase in the transition amplitude and to a super-high-resolution spectroscopy.

Experiments of coherent laser beams can be divided into two groups. In the first one, atoms or molecules interact with parallel and spatially separated laser beams. The atomic and molecular transitions occur at spatially separated regions and completely different times. The external phase is introduced into the coherent transition amplitude through the displacement of the entire laser-atom or molecular system. The saturation experiment of separated laser beams was first studied by Baklanov et al. ${ }^{2}$ and has been beautifully demonstrated by Bergquist et al. ${ }^{3}$ The experiment of separated laser beams, free of Doppler effect, for two-photon absorption was proposed by Baklanov et al. ${ }^{4}$ The latter experiment has been performed by Chebotayev et al..$^{5}$ and Helmcke et al. ${ }^{6}$ The separated-beam experiment of Raman scattering has recently been proposed by Chebotayev et al. ${ }^{7}$ The experiments of this group lead to super-high-resolution spectroscopy.

In the second group, atoms or molecules interact with crossed-laser beams. The atomic or molecular transitions occur at the same spatial region. The external phase is introduced into the coherent transition amplitude through the displacement of a part of the quantum system; namely the laser, while the atoms or molecules are un- affected. In the experiment proposed by $\mathrm{Li}^{1}$ on the Doppler-free two-photon absorption of two coherent beams, the atomic beam passes through the intersection of crossed-laser beams. The proposed experiment can lead to the direct phase determination of the transition amplitude. In the crossed-beam experiments performed by Teets et al. and Salour et al., ${ }^{8}$ the interest was centered solely on the superfine spectral resolution in the presence of laser pulse broadening.

There are several approaches to the theoretical discussion of the atomic and molecular absorption initiated by coherent laser beams. Baklanov, Chebotayev, Dubetsky, and co-workers ${ }^{4,5,7}$ used two-level or three-level dynamics with a weak field approximation. Bordé and co-workers ${ }^{9}$ used the density matrix formulation with the help of perturbative and numerical methods. It was pointed out by $\mathrm{Li}^{10}$ that the main features on the absorption process initiated by coherent laser beams do not depend on detail dynamics, but follow directly from basic quantum mechanics.

In the separated-beam experiment, the experimental arrangement is important; however, such an importance has not been recognized before. In this paper, three different arrangements for the separated-beam experiment of the Doppler-free two-photon absorption are considered. These arrangements lead to different Ramsey fringe variations. In the first one, the Ramsey fringe cannot be observed. While in the second, the Ramsey fringe variation not only depends on the atom's time of flight, but also the transit-time of atoms crossing a laser beam. These two cases are discussed in Sec. II. Finally in the third, the transit-time broadening is eliminated; and the true Ramsey fringe variation, which depends only on the atom's time of flight is observed. This case is presented in Sec. III. In Sec. IV, the sep- 
arated-beam experiment of the saturated absorption is duscussed. It shows that even in the presence of transit-time broadening, the Ramsey fringe variation depends only on the atom's time of flight. In Sec. V, some theoretical aspects of experiments of separated beams that had been performed will be discussed.

\section{TRANSITION AMPLITUDE}

In a separated-beam experiment, an atomic beam intersects with two coherent and spatially separated laser beams. A simple experimental arrangement is depicted in Fig. 1. When the atomic beam enters the first intersection region, some of the atoms absorb a pair of opposite-momentum photons and become excited. After the absorption, the atom's momentum remains unchanged. The excited and unexcited atoms move together at the same velocity. Upon entering the second intersection region, some of the atoms again absorb a pair of opposite-momentum photons and become excited. These excited atoms mix with the previously excited atoms and move ahead with the rest of the atoms. The fluorescence from the excited atoms is measured by detector $D$. Since it is impossible for detector $D$ to distinguish the fluorescence emitted by the excited atoms from the first intersection region and those of the second intersection region, a direct interference phenomenon is thus recorded.

The Doppler-free two-photon transition amplitude for the atomic absorption induced by the laser beams in the first intersection region can be written as

$$
g\left(\omega_{0}\right)\left\langle\overrightarrow{\mathrm{p}}, \epsilon_{2}|t| \overrightarrow{\mathrm{p}}, \epsilon_{1} ; \overrightarrow{\mathrm{k}},-\overrightarrow{\mathrm{k}}\right\rangle,
$$

where $g\left(\omega_{0}\right)$ describes the atomic line profile. ${ }^{11}$ $\left\langle\overrightarrow{\mathrm{p}} ; \epsilon_{2}|t| \overrightarrow{\mathrm{p}}, \epsilon_{1} ; \overrightarrow{\mathrm{k}},-\overrightarrow{\mathrm{k}}\right\rangle$ is the reduced transition amplitude. In the transition, the momentum $\vec{p}$ of the

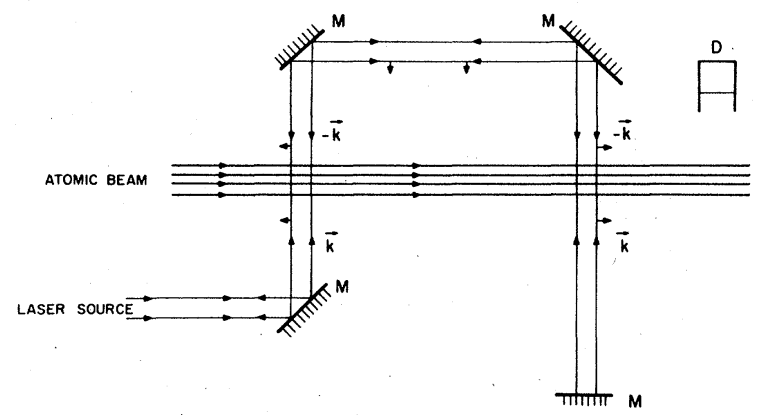

FIG. 1. Separated-beam experiment of a Dopplerfree two-photon absorption for which the Ramsey interference fringes cannot be observed. $M$ denotes reflecting mirrors. The fluorescence from the excited atoms is measured by the detector $D$. atom remains the same, while its internal energy is changed from $\epsilon_{1}$ to $\epsilon_{2}$,

$$
\epsilon_{2}-\epsilon_{1}=\hbar \omega_{0} \text {. }
$$

The wave vectors $\vec{k}$ and $-\vec{k}$ for the incident and reflected laser beams are indicated in Fig. 1 with

$$
|\vec{k}|=k=\omega / c,
$$

where $c$ is the velocity of light. The atomic absorption induced in the second intersection region occurred at a later time $T$ as compared with that from the first region. The corresponding transition amplitude has the form

$$
g\left(\omega_{0}\right)\left\langle\overrightarrow{\mathrm{p}}, \epsilon_{2}\left|e^{i T H / \hbar} t e^{-i T H / \hbar}\right| \overrightarrow{\mathrm{p}}, \epsilon_{1} ; \overrightarrow{\mathrm{k}},-\overrightarrow{\mathrm{k}}\right\rangle,
$$

where $H$ is the total Hamiltonian of the whole quantum system and $T$ the time of flight for the atomic beam between two spatially separated laser beams. Since the detector is unable to distinguish the absorptions from the two laser beams, the amplitudes in Eqs. (1.1) and (1.4) have to be added coherently :

$$
\begin{aligned}
& g\left(\omega_{0}\right)\left(\left\langle\overrightarrow{\mathrm{p}}, \epsilon_{2}|t| \overrightarrow{\mathrm{p}}, \epsilon_{1} ; \overrightarrow{\mathrm{k}},-\overrightarrow{\mathrm{k}}\right\rangle\right. \\
& \left.\quad+\left\langle\overrightarrow{\mathrm{p}}, \epsilon_{2}\left|e^{i T H / \hbar} t e^{-i T H / \hbar}\right| \overrightarrow{\mathrm{p}}, \epsilon_{1} ; \overrightarrow{\mathrm{k}},-\overrightarrow{\mathrm{k}}\right\rangle\right)
\end{aligned}
$$

If the laser beams are truly well defined, that is to say, their wave vectors are exactly equal to $\vec{k}$ and $-\vec{k}$ with no uncertainty, then Eq. (1.5) can be written as

$$
g\left(\omega_{0}\right)\left\langle\overrightarrow{\mathrm{p}}, \epsilon_{2}|t| \overrightarrow{\mathrm{p}}, \epsilon_{1} ; \overrightarrow{\mathrm{k}},-\overrightarrow{\mathrm{k}}\right\rangle\left\{1+\exp \left[i\left(\omega_{0}-2 \omega\right) T\right]\right\} .
$$

The transition amplitude in Eq. (1.6) depends on the external phase $\left(\omega_{0}-2 \omega\right) T$, which can be varied directly be either chaning the laser-beam frequency $\omega$ or the time of flight $T$. The resonance fringes induced by these variations are referred to as optical Ramsey fringes. ${ }^{2}$

Upon arriving at Eq. (1.6) one has not considered the finite-width effect of the laser beam. The finiteness leads to the uncertainty of the wave vector $\overrightarrow{\mathrm{k}}$, which will modify the spectral profile and Ramsey fringe. This problem will be dealt with in the following sections.

\section{TRANSIT-TIME BROADENING}

The finite width of a laser beam leads to a broadening effect ${ }^{12}$ in the super-high-resolution spectral lines. This effect is usually referred to as the transit-time broadening. In the separatedbeam experiment of the Doppler-free two-photon absorption, the broadening effect depends highly on the experimental arrangement. Only the broadening effect for experiments with simpler geometrical arrangements is examined here. 
Elimination of interference

The existence of wave-vector uncertainty due to the finite width of a laser beam may eliminate interference in the separated-beam experiment. To show this, let us return to the experiment in Fig. 1. For instance, if the wave vector of the initial laser beam before crossing the atomic beam has an uncertain transverse component in the opposite direction from the atomic velocity, then all other reflected beams also have uncertain components, and having directions as indicated in Fig. 1 by small arrows. The separated beams on the right have components in the same direction and the left in the opposite direction from the atomic velocity. Those atoms absorbing the photon pair in the left intersection region slowed down, while, on the other hand, those atoms absorbing the photon pair in the right intersection region were accelerated. Hence, the excited

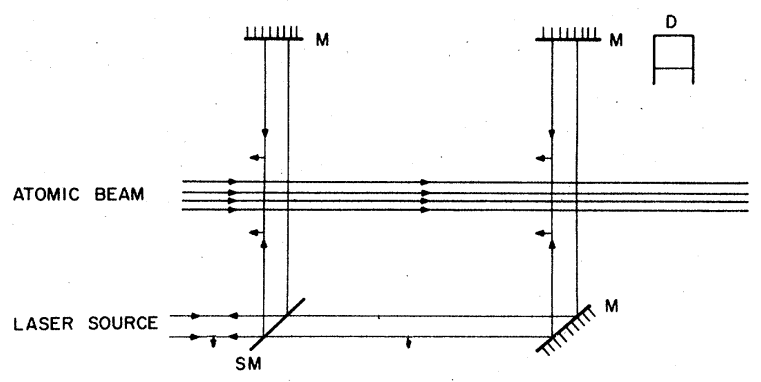

FIG. 2. Separated-beam experiment with a coherent transit-time broadening. SM is the laser-beam splitter

atoms from these two regions can be distinguished by their velocity. The atomic absorption from the spatially separated beams is no longer coherent and the resonance fringe variation cannot be observed through detector $D$.

Coherent broadening

Consider the experiment depicted in Fig. 2. Now, if one of the laser beams has an uncertain transverse wave-vector component in the opposite direction of the atomic velocity, then all separated beams have the components in the opposite direction. After absorbing the photon pair, the excited atoms from both the first and second intersection regions will slow down. These atoms are no longer distinguishable and the resonance fringe variations will be recorded by detector $D$.

In the presence of the transverse components, the coherent amplitude in Eq. (15) should be rewritten as

$$
\begin{aligned}
g\left(\omega_{0}\right)\left(\left\langle\overrightarrow{\mathrm{p}}, \epsilon_{2}|t| \overrightarrow{\mathrm{p}}, \epsilon_{1} ; \overrightarrow{\mathrm{k}}+\overrightarrow{\mathrm{k}}_{x},-\overrightarrow{\mathrm{k}}+\overrightarrow{\mathrm{k}}_{x}\right\rangle+\right. & \left.\left\langle\overrightarrow{\mathrm{p}}, \epsilon_{2}\left|e^{i T H / \hbar} t e^{-i T H / \hbar}\right| \overrightarrow{\mathrm{p}}, \epsilon_{1} ; \overrightarrow{\mathrm{k}}+\overrightarrow{\mathrm{k}}_{x},-\overrightarrow{\mathrm{k}}+\overrightarrow{\mathrm{k}}_{x}\right\rangle\right) \\
& \left.=g\left(\omega_{0}\right)\left\langle\overrightarrow{\mathrm{p}}, \epsilon_{2}|t| \overrightarrow{\mathrm{p}}, \epsilon_{1} ; \overrightarrow{\mathrm{k}}+\overrightarrow{\mathrm{k}}_{x},-\overrightarrow{\mathrm{k}}+\overrightarrow{\mathrm{k}}_{x}\right)\left\{1+\exp \left[i\left(\omega_{0}-2 \omega\right) T+2 i v k_{x} T\right)\right]\right\},
\end{aligned}
$$

where $v$ is the velocity of the atomic beam and $k_{x}$ is the wave-vector component of the separated parallel laser beams in the direction of the atomic velocity. In most cases, the dimension of the laser-beam width is much larger than its wavelength. Hence,

$$
k_{x} \ll k \text {. }
$$

In reaching the last step of Eq. (2.1), high-order Doppler-shift contributions have been neglected, and only the first-order contribution is kept.

From Eq. (2.1), one can obtain the transition probability

$$
\int d k_{x}^{\prime} G^{2}\left(k_{x}^{\prime}\right)\left|g\left(\omega_{0}\right)\left\langle\overrightarrow{\mathrm{p}}, \epsilon_{2}|t| \overrightarrow{\mathrm{p}}, \epsilon_{1} ; \overrightarrow{\mathrm{k}}+\overrightarrow{\mathrm{k}}_{x}^{\prime},-\overrightarrow{\mathrm{k}}+\overrightarrow{\mathrm{k}}_{x}^{\prime}\right\rangle\left\{1+\exp \left[i\left(\omega_{0}-2 \omega\right) T+2 i v k_{x}^{\prime} T\right]\right\}\right|^{2},
$$

where $G\left(k_{x}^{\prime}\right)$ describes the transverse profile of the laser beam. To simplify the calculation, functions $G\left(k_{x}^{\prime}\right)$ and $g\left(\omega_{0}\right)$ are assumed to have Gaussian shapes

$$
G\left(k_{x}^{\prime}\right)=\exp \left(-A{k_{x}^{\prime 2}}^{2}\right)
$$

and

$$
g\left(\omega_{0}\right)=\exp \left[-\frac{B}{\hbar^{2}}\left(\epsilon_{2}+\frac{1}{2 m}\left(\overrightarrow{\mathrm{p}}+2 \hbar \overrightarrow{\mathrm{k}}_{x}^{\prime}\right)^{2}-\epsilon_{1}-\frac{1}{2 m} \overrightarrow{\mathrm{p}}^{2}-2 \hbar c\left(k^{2}+k_{x}^{\prime 2}\right)^{1 / 2}\right)\right],
$$

where $m$ is the atomic mass. Constant $A$ is related to the laser-beam width and constant $B$ is related to the absorption line width in the absence of the transit-time broadening. Under the lowest-order approximation with respect to the uncertain momentum $\overrightarrow{\mathrm{k}}_{x}$, Eq. (2.5) is simplified to

$$
g\left(\omega_{0}\right)=\exp \left[-B\left(\omega_{0}-2 \omega+2 v k_{x}^{\prime}\right)^{2}\right] .
$$

After performing the integration in Eq. (2.3) and eliminating irrelevant factors, one has 


$$
\left|\left\langle\overrightarrow{\mathrm{p}}, \epsilon_{2}|t| \overrightarrow{\mathrm{p}}, \epsilon_{1} ; \overrightarrow{\mathrm{k}},-\overrightarrow{\mathrm{k}}\right\rangle\right|^{2} \exp \left(-\left(\dot{\omega}_{0}-2 \omega\right)^{2} \frac{2 A B}{A+4 B v^{2}}\right)\left\{1+\cos \left(\frac{T\left(\omega_{0}-2 \omega\right) A}{A+4 B v^{2}}\right) \exp \left[-\frac{T^{2}}{8 B}\left(1-\frac{A}{A+4 B v^{2}}\right)\right]\right\}
$$

In reaching Eq. (2.7) it is assumed that the reduced transition amplitude is a slowly varying function of the wave vector $\vec{k}$. The first exponential factor in Eq. (2.7) denotes the spectral line shape of the Dopplerfree two-photon absorption in one laser beam under the influence of the transit-time broadening. The resonance fringe variation

$$
\cos \left(\frac{T\left(\omega_{0}-2 \omega\right) A}{A+4 B v^{2}}\right)
$$

has a fringe spacing

$$
\frac{1}{2 T}\left(1+\frac{4 B v^{2}}{A}\right)
$$

In the absence of the transverse uncertainty, the optical Ramsey fringe spacing for the Doppler-free twophoton absorption from Eq. (1.6) has the fringe spacing $1 / 2 T$. In the present case, the presence of the transverse uncertainty not only causes the transit-time broadening of the spectral line shape, but also the broadening of the Ramsey fringe spacing as expressed in Eq. (2.9). Hence, the Ramsey fringe spacing appearing in the Doppler-free two-photon absorption depends on the experimental arrangement.

Incoherent broadening

The experiment is depicted in Fig. 3. Here two identical laser sources are used. During a Dopplerfree two-photon absorption, the atom absorbs a photon from each of these sources. Since the two photons are independent of each other, their momentum uncertainties are unrelated. Equation (2.3) should be modified to

$$
\iint d k_{x}^{\prime} d k_{x}^{\prime \prime} G_{1}\left(k_{x}^{\prime}\right) G_{2}\left(k_{x}^{\prime \prime}\right) \mid g\left(\omega_{0}\right)\left\langle\overrightarrow{\mathrm{p}}, \epsilon_{2}|t| \overrightarrow{\mathrm{p}}, \epsilon_{1} ; \overrightarrow{\mathrm{k}}+\overrightarrow{\mathrm{k}}_{x}^{\prime},-\overrightarrow{\mathrm{k}}+\left.\overrightarrow{\mathrm{k}}_{x}^{\prime \prime}\left\{1+\exp \left[i\left(\omega_{0}-2 \omega\right) T+i v\left(k_{x}^{\prime}+k_{x}^{\prime \prime}\right) T\right]\right\}\right|^{2}\right.
$$

with

$$
\begin{aligned}
& G_{1}\left(k_{x}^{\prime}\right)=\exp \left(-A_{1}{k_{x}^{\prime}}^{2}\right), \\
& G_{2}\left(k_{x}^{\prime \prime}\right)=\exp \left(-A_{2} k_{x}^{\prime \prime 2}\right),
\end{aligned}
$$

and

$$
g\left(\omega_{0}\right)=\exp \left[-B\left(\omega_{0}-2 \omega+v k_{x}^{\prime}+v k_{x}^{\prime \prime}\right)^{2}\right]
$$

The constants $A_{1}$ and $A_{2}$ are related to beam widths of laser sources I and II, respectively. After performing the integrations in Eq. (2.10) and eliminating irrelevant factors, one has

$$
\begin{aligned}
& \left|\left\langle\overrightarrow{\mathrm{p}}, \epsilon_{2}|t| \overrightarrow{\mathrm{p}}, \epsilon_{1} ; \overrightarrow{\mathrm{k}},-\overrightarrow{\mathrm{k}}\right\rangle\right|^{2} \exp \left(-\left(\omega_{0}-2 \omega\right)^{2} \frac{2 A_{1} A_{2} B}{A_{1} A_{2}+2\left(A_{1}+A_{2}\right) B v^{2}}\right) \\
& \quad \times\left\{1+\cos \left(\frac{T\left(\omega_{0}-2 \omega\right) A_{1} A_{2}}{A_{1} A_{2}+2\left(A_{1}+A_{2}\right) B v^{2}}\right) \exp \left[-\frac{T^{2}}{8 B}\left(1-\frac{A_{1} A_{2} B}{A_{1} A_{2}+2\left(A_{1}+A_{2}\right) B v^{2}}\right)\right]\right\} .
\end{aligned}
$$

The corresponding broadened linewidth and Ramsey fringe spacing can be obtained from Eq. (2.14). The following point is worth noting; if the laser beams from two independent sources have the same width $A_{1}=A_{2}$, then Eqs. (2.7) and (2.14) are essentially the same. Hence there is no difference in using one or two laser sources to initiate the separated-beam experiment of the Dopplerfree two-photon absorption with a transit-time broadening.

\section{ELIMINATION OF TRANSIT-TIME BROADENING}

Through a simple alteration, the transit-time broadening can be eliminated in the Doppler-free two-photon absorption. This can be achieved by inserting a lens into the experimental setup in Fig. 1. The new arrangement is depicted in Fig. 4. The last reflecting mirror is on the focal plane of the converging lens. The lens and mirror combination inverts the uncertain transverse compo- 


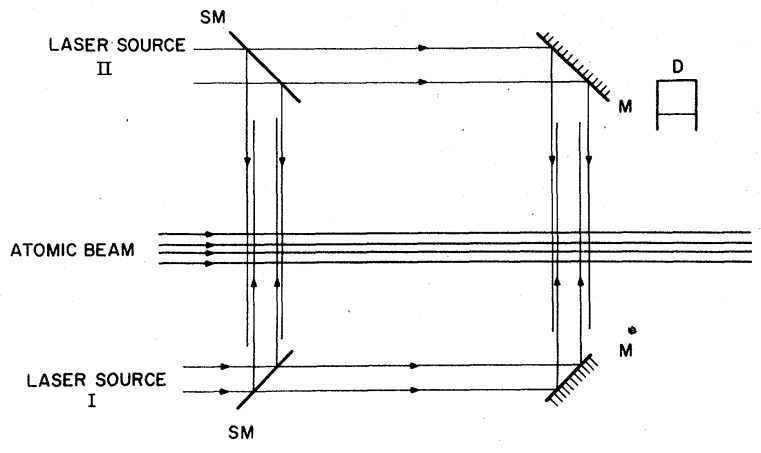

FIG. 3. Separated-beam experiment with an incoherent transit-time broadening.

nent of the wave vector after the reflection. The relative orientations of these components in the initial and reflected laser beams are indicated by small vectors in Fig. 4. Now during a Dopplerfree two-photon absorption, the transverse wavevector uncertainty will not effect the atomic velocity. The transition probability can be written as

$$
\begin{aligned}
\int d k_{x}^{\prime} G^{2}\left(k_{x}^{\prime}\right) & \mid g\left(\omega_{0}\right)\left\langle\overrightarrow{\mathrm{p}}, \epsilon_{2}|t| \overrightarrow{\mathrm{p}}, \epsilon_{1} ; \overrightarrow{\mathrm{k}}+\overrightarrow{\mathrm{k}}_{x}^{\prime},-\overrightarrow{\mathrm{k}}-\overrightarrow{\mathrm{k}}_{x}^{\prime}\right\rangle \\
\times & \times\left.\left\{1+\exp \left[i\left(\omega_{0}-2 \omega\right) T\right]\right\}\right|^{2}
\end{aligned}
$$

with

$$
g\left(\omega_{0}\right)=\exp \left[-B\left(\omega_{0}-2 \omega\right)^{2}\right] .
$$

The Ramsey phase in Eq. (3.1) and the line shape in Eq. (3.2) are no longer dependent on the transverse uncertainty of the wave vector. Equation (3.1) is simply proportional to

$$
\left|g\left(\omega_{0}\right)\left\langle\overrightarrow{\mathrm{p}}, \epsilon_{2}|t| \overrightarrow{\mathrm{p}}, \epsilon_{1} ; \overrightarrow{\mathrm{k}},-\overrightarrow{\mathrm{k}}\right\rangle\right|{ }^{2}\left[1+\cos \left(\omega_{0}-2 \omega\right) T\right] .
$$

Hence in the present case the transit-time broadening appears neither in the spectral line nor in Ramsey fringes. The fringe spacing is given by $1 / 2 T$. This is the result commonly cited in most

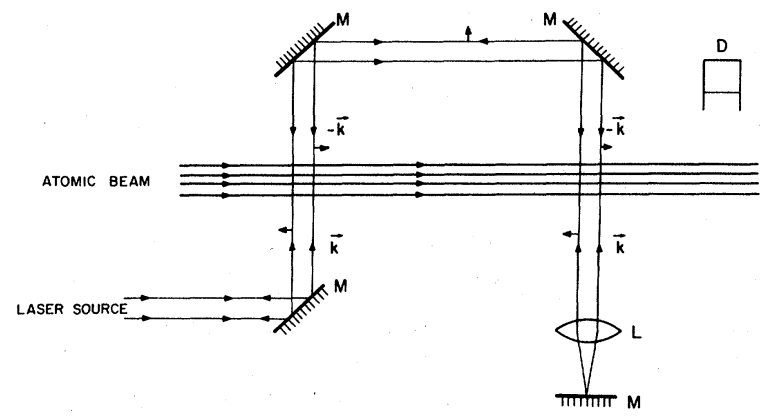

FIG. 4. Separated-beam experiment without a transittime broadening. The end mirror is on the focal plane of the lens $L$. published articles.

Beside the transit-time broadening, the other finite-beam-width effect is the diffraction loss in the resonance cavity of gas lasers. The resonance cavity with plane-parallel mirrors is largely of historical interest. In this geometrical arrangement, the uncertain transverse wave vector of light in the cavity is neither inverted nor becomes smaller upon the reflection from plane mirrors. The beam increases its width after each reflection and has a high diffraction loss. The resonance cavity with confocal spherical mirrors is used in practice. In the latter arrangement, upon the reflection from spherical mirrors the uncertain transverse wave vector becomes smaller, which in turn lowers the increasing rate of the beam width and reduces the diffraction loss greatly. Hence the diffraction loss, resulting from the finite beam width of the oppositely propagated beams in the resonance cavity, is highly dependent on the experimental arrangement. The discussions presented in Secs. II and III demonstrated that the transit-time broadening effect of the Doppler-free two-photon absorption, which also resulted from the finite beam width of the oppositely propagated beams, resembles the above diffraction loss and is also highly dependent on the experimental arrangement.

Our experience toward the transit-line broadening in the atomic absorption is mainly from the one-photon process. The transit-time broadening effect in the two-photon process has not been given clearly in the literature. For example, in the discussion given by Baklanov, Dubetsky, and Chebotayev $^{4}$ the two-photon absorption process is transittime limited. However, their final result only exhibits a Ramsey fringe variation. The transit-time broadening effect cannot be traced from their formulation. Since to understand the relation among the transit-time broadening and the Ramsey fringe variation, a new theoretical explanation should be adopted.

To observe the Doppler-free two-photon absorption, experimenters had taken a great caution in handling the reflected beam. Usually a concaved mirror or a lens and plane-mirror combination was used as a reflector. This is, of course, the mechanism to minimize the transit-time broadening.

On the discussion presented above, the secondorder Doppler effect has not been included. Its inclusion would lead to an interesting distortion of the Ramsey fringes. ${ }^{13}$ The effect of the optical system misalignment is also not included, which would also lead to a similar distortion. The important message in this section is that it is the experiment arrangement and not the beam sep- 
aration for overcoming the transit-time broadening in the Doppler-free two-photon absorption.

\section{SATURATED ABSORPTION}

In this section we shall discuss the saturated absorption phenomenon with spatially separated laser beams. To achieve such an absorption, a number of experimental arrangements are possible. For our purpose, the discussion is only concerned with the three-beam experiment as performed by Bergquist et al. ${ }^{3}$ Their experimental arrangement is depicted in Fig. 5. A reflecting mirror is located on the focal planes of each converging lens. Laser beams have the same width. Their relative transverse uncertain components are indicated by small arrows. The spatial separations between parallel laser beams are equal and the atomic beam is perpendicular to these laser beams.

Let us consider a single-photon absorption process, with atomic beam perpendicularly intersecting with two parallel and coherent laser beams with a wave vector $\overrightarrow{\mathrm{k}}$ and transverse uncertain component $\overrightarrow{\mathrm{k}}_{x}$. The transition amplitude $F\left(\vec{k}_{,} \overrightarrow{\mathrm{k}}_{x}\right)$ can be written as

$$
\begin{gathered}
F\left(\overrightarrow{\mathrm{k}}, \overrightarrow{\mathrm{k}}_{x}\right)=g\left(\omega_{0}\right)\left[\left\langle\overrightarrow{\mathrm{p}}+\left(\overrightarrow{\mathrm{k}}+\overrightarrow{\mathrm{k}}_{x}\right), \epsilon_{2}|t| \overrightarrow{\mathrm{p}}, \epsilon_{1} ; \overrightarrow{\mathrm{k}}+\overrightarrow{\mathrm{k}}_{x}\right\rangle\right. \\
+\left\langle\overrightarrow{\mathrm{p}}+\left(\overrightarrow{\mathrm{k}}+\overrightarrow{\mathrm{k}}_{x}\right), \epsilon_{2}\left|e^{i T H / \hbar} t e^{-i T H / \hbar}\right| \overrightarrow{\mathrm{p}}\right. \\
\left.\left.\epsilon_{1} ; \overrightarrow{\mathrm{k}}+\overrightarrow{\mathrm{k}}_{x}\right\rangle\right]
\end{gathered}
$$

The notations used here are the same as those in the preceding sections. When high-order Dopplershift contributions are neglected, Eq. (4.1) has the form

$$
\begin{aligned}
F\left(\vec{k}_{x}\right)= & g\left(\omega_{0}\right)\left\langle\overrightarrow{\mathrm{p}}+\left(\overrightarrow{\mathrm{k}}+\overrightarrow{\mathrm{k}}_{x}\right), \epsilon_{2}|t| \overrightarrow{\mathrm{p}}, \epsilon_{1} ; \overrightarrow{\mathrm{k}}+\overrightarrow{\mathrm{k}}_{x}\right\rangle \\
& \times\left\{1+\exp \left[i\left(\omega_{0}-\omega\right) T+i\left(k v_{z}+k_{x} v\right) T\right]\right\},
\end{aligned}
$$

with

$$
g\left(\omega_{0}\right)=\exp \left[-B\left(\omega_{0}-\omega+k v_{z}+k_{x} v\right)^{2}\right]
$$

In Eq. (4.2), a term depending on the transverse velocity of the atomic beam is included. Experimentally, even a well-collimated atomic beam contains a distribution of transverse velocity. The atomic velocity $v$ is perpendicular to the wave vector $\overrightarrow{\mathrm{k}}$ of parallel laser beams. When $N$ number of atoms in the atomic beam cross laser beams, only a portion of them become excited. The excited atoms can be expressed as

$$
N D\left|F\left(\overrightarrow{\mathrm{k}}, \overrightarrow{\mathrm{k}}_{x}\right)\right|^{2}
$$

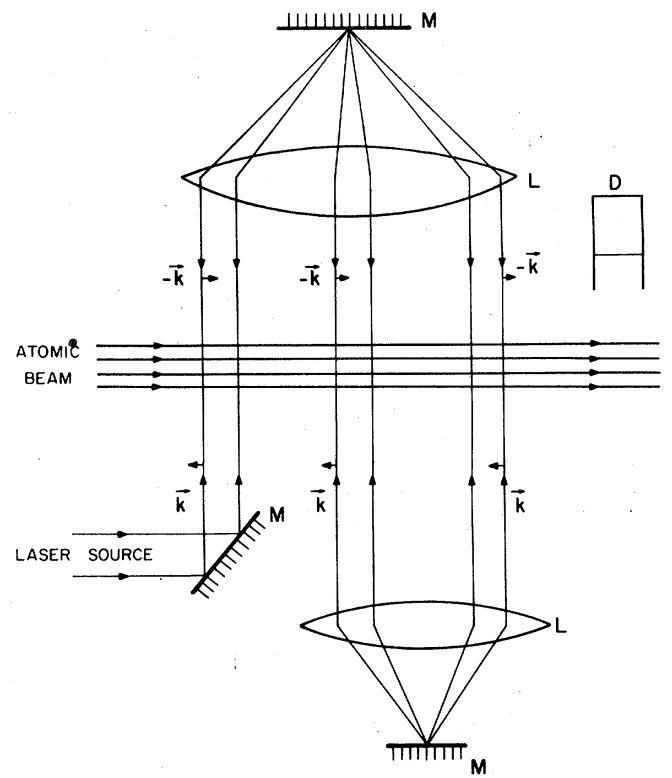

FIG. 5. Three-zone separated-beam experiment of a saturated absorption.

The rest of the atoms are

$$
N\left[1-D\left|F\left(\vec{k}, \overrightarrow{\mathrm{k}}_{x}\right)\right|^{2}\right],
$$

where the constant $D$ is related to the laser-beam intensity.

From Eqs. (4.2), (4.4), and (4.5) it is clear that the spatial separation between laser beams has induced a phase variation to the populations of excited and unexcited atoms. However, the variations not only depend on the time of flight of atoms, but also on the transverse velocity of atoms. To obtain the experimentally observed quantities, one has to perform an average with respect to the distribution of the atomic transverse velocity. The average will eliminate or obscure the variations.

To overcome the above difficulty in observing variations, one can excite those unexcited atoms by another pair of coherent parallel laser beams with the wave vector $-\vec{k}$ and transverse uncertain component $-\overrightarrow{\mathrm{k}}_{x}$. The spatial separations between the latter pair is the same as that of the former pair. After crossing these two pairs of laser beams the number of excited atoms is then

$$
\begin{aligned}
& N D|F(\mathrm{k}, \overrightarrow{\mathrm{k}})|^{2}+N D\left|F\left(-\overrightarrow{\mathrm{k}},-\overrightarrow{\mathrm{k}}_{x}\right)\right|^{2} \\
&-N D^{2}\left|F\left(\overrightarrow{\mathrm{k}}_{,} \overrightarrow{\mathrm{k}}_{x}\right) F\left(-\overrightarrow{\mathrm{k}},-\overrightarrow{\mathrm{k}}_{x}\right)\right|^{2} .
\end{aligned}
$$

With the help of Eq. (4.2), one can rewrite Eq. (4.6) as 


$$
\begin{aligned}
2 N D g^{2}\left(\omega_{0}\right)\left|\left\langle\overrightarrow{\mathrm{p}}+\left(\overrightarrow{\mathrm{k}}+\overrightarrow{\mathrm{k}}_{x}\right), \epsilon_{2}|t| \overrightarrow{\mathrm{p}}, \epsilon_{1} ; \overrightarrow{\mathrm{k}}+\overrightarrow{\mathrm{k}}_{x}\right\rangle\right| 2\left\{1+\cos \left[\left(\omega_{0}-\omega\right) T+\left(k v_{z}+k_{x} v\right) T\right]\right\} \\
+2 N D g^{2}\left(\omega_{0}\right)\left|\left\langle\overrightarrow{\mathrm{p}}-\left(\overrightarrow{\mathrm{k}}+\overrightarrow{\mathrm{k}}_{x}\right), \epsilon_{2}|t| \overrightarrow{\mathrm{p}}, \epsilon_{1} ;-\overrightarrow{\mathrm{k}}-\overrightarrow{\mathrm{k}}_{x}\right\rangle\right|^{2}\left\{1+\cos \left[\left(\omega_{0}-\omega\right) T-\left(k v_{z}+k_{x} v\right) T\right]\right\} \\
-4 N D^{2} g^{2}\left(\omega_{0}\right) g_{0}^{2}\left(\omega_{0}\right)\left|\left\langle\overrightarrow{\mathrm{p}}+\left(\overrightarrow{\mathrm{k}}+\overrightarrow{\mathrm{k}}_{x}\right), \epsilon_{2}|t| \overrightarrow{\mathrm{p}}, \epsilon_{1} ; \overrightarrow{\mathrm{k}}+\overrightarrow{\mathrm{k}}_{x}\right\rangle\right|^{2}\left|\left\langle\overrightarrow{\mathrm{p}}-\left(\overrightarrow{\mathrm{k}}+\overrightarrow{\mathrm{k}}_{x}\right), \epsilon_{2}|t| \overrightarrow{\mathrm{p}}, \epsilon_{1} ;-\overrightarrow{\mathrm{k}}-\overrightarrow{\mathrm{k}}_{x}\right\rangle\right|^{2} \\
\quad \times\left\{1+2 \cos \left[\left(\omega_{0}-\omega\right) T\right] \cos \left[\left(k v_{z}+k_{x} v\right) T\right]+\frac{1}{2} \cos \left[2\left(k v_{z}+k_{x} v\right) T\right]+\frac{1}{2} \cos \left[2\left(\omega_{0}-\omega\right) T\right]\right\}
\end{aligned}
$$

where

$$
g_{1}(\omega)=\exp \left[-B\left(\omega_{0}-\omega-k v_{z}-k_{x} v\right)^{2}\right] .
$$

There are a number of variational terms in Eq. (4.7). Except for the last term, the others all depend on the transverse velocity and will be eliminated after the average with respect to the distribution of the atomic transverse velocity. This last term is often referred to as the Ramsey fringe variation.

The Ramsey fringe variation in Eq. (4.7) does not depend on the spatial separation between laser pairs. One may overlay two laser beams with one each from two laser pairs. The three-beam experiment in Fig. 5 is such a realization.

\section{DISCUSSION}

It has been shown in Secs. II and III that the Ramsey fringe variation appearing in the Doppler-free two-photon absorption of the separated-beam experiment depended on the experimental arrangement. With the arrangement as shown in Fig. 1, the separated-beam experiment does not lead to Ramsey fringe variation, but with those in Figs. 2 and 3 , the transit-time broadening is present, and the Ramsey fringe variations not only depend on the time of flight, but also the transit-time broadening. However, with the arrangement in Fig. 4, the transit-time broadening is eliminated, and the Ramsey fringe variations now depend only on the time of flight. The situation in the saturated absorption is quite different, as it is shown in Sec. IV that even in the presence of the transittime broadening the Ramsey fringe variations depend only on the time of flight.

In the performed experiments on the Dopplerfree two-photon absorption of separated beams, the experimental arrangements differ from those discussed in the previous section. In the experiment of Chebotayev, Shishayev, Yurshin, and Vasilenko, ${ }^{5}$ a lens-mirror combination is used; if the mirror is placed on the focal plane of the lens, then after the reflection the uncertain transverse component of the incident light wave vector would be inverted. Their experiment would have been a separated-beam experiment of the Doppler-free two-photon absorption without the transit-time broadening. From Eq. (3.3), the Ramsey fringe spacing for such an experiment is $1 / 2 T$. The time of flight in their experiment is $T=1.3 \times 10^{-7} \mathrm{sec}$. which corresponds to the fringe spacing of 3.8 $\mathrm{MHz}$. However, in their published article, they did not mention whether or not the mirror is located on the focal plane of the lens. Since the fringe spacing which they observed is $5.7 \pm 3.5$ $\mathrm{MHz}$, it indicates that the mirror probably is not located on the focal plane.

As the mirror moves away from the focal plane, the inversion of the uncertain transverse component becomes less complete and the transit-time effects gradually appear. Its appearance will increase the Ramsey fringe spacing. If the uncertain transverse component is not inverted or the uncertain transverse components of two counter propagating light waves are not correlated, the Ramsey fringe spacing from Eqs. (2.9) and (2.14) can be expressed as

$$
\frac{1}{2 T}\left(1+\frac{2 t^{2}}{t_{t}^{2}}\right)
$$

where $t$ is the lifetime of the excited state and $t_{t}$ the transit-time of atoms crossing the laser beam. Then the fringe space for the above-mentioned experiment would be $8 \mathrm{MHz}$. In comparison with the experimentally observed value $5.7 \pm 3.5 \mathrm{MHz}$, one might assume that the transit-time broadening in the said experiment has been reduced in some way. In the experiment of Helmcke, Lee, and Hall, ${ }^{6}$ a folded resonator is used. The end mirror in the resonator reduces the uncertain transverse components after the reflection. Otherwise the resonance would not occur in the resonator. If their experiment can be considered as a separated-beam experiment without the transit-time broadening, the expected fringe spacing would be $1 / 2 T=70$ $\mathrm{kHz}$, which is somewhat less than the estimated experimental value of $100 \mathrm{kHz}$. This is due to the small residual transverse component of the absorbed photon pair. However, they attributed the above discrepancy to the laser frequency noise.

In the separated-beam experiment of a saturated absorption, ${ }^{3}$ a pair of well-corrected cat's-eye retroreflectors are used. The experimental arrangement is similar to that in Fig. 5. Although the transit-time broadening effect exists in the saturated absorption as seen from Eqs. (4.3) and 
(4.8). The Ramsey fringe spacing depends only on the time of flight and is equal to $1 / 2 T$, which has been confirmed by the experiment.

The interference phenomena of one-photon and two-photon absorption processes are distinctively different. They are represented by Young's double-slit and Brown-Twist's experiments, respectively. The recognition of such a difference has led to the development of quantum optics. The saturated absorption of separated beams is a coherent one-photon absorption process, in which there is no two-photon correlation effect. On the other hand, the Doppler-free two-photon absorption of separated beams is a coherent two-photon absorption process, in which there is a two-photon correlation effect. However, such an important effect has not been recognized in the literature.
On the present work, the uncertain momenta of the absorbed photon pair in the Doppler-free twophoton absorption are correlated. The correlation has made Ramsey interference fringes in these one-photon and two-photon processes distinctively different. For the saturated absorption, the fringe variation depends on the time of atomic flight alone. For the Doppler-free two-photon absorption, the dependence is much more complicated and a beam separation does not necessarily lead to the observation of Ramsey interference fringes.

\section{ACKNOWLEDGMENT}

The author is grateful to Dr. J. L. Hall, Dr. R. L. Barger, and Professor J. A. Jacobs for numerous interesting discussions.
${ }^{1}$ Ming Chiang Li, Nuovo Cimento 39B, 165 (1977).

${ }^{2}$ Ye. V. Baklanov, B. Ya. Dubetsky, and V. P. Chebotayev, Appl. Phys. 9, 171 (1976); 11, 201 (1976); B. Ya. Dubetsky, Kvant. Elektron. (Moscow) $\underline{3}, 1189$ (1976) [Sov. J. Quantum Electron. 6, 682 (1976)].

${ }^{3}$ J. C. Bergquist, S. A. Lee, and J. $\bar{L}$. Hall, Phys. Rev. Lett. 38, 159 (1977); in Laser Spectroscopy III, edited by J. L. Hall and J. L. Carlsten (Springer, Berlin, 1977), Vol. 7, p. 142; R. L. Barger, J. C. Bergquist, J. C. English, and D. J. Glaze, Appl. Phys. Lett. 34, 850 (1979).

${ }^{4}$ Ye. V. Baklanov, V. P. Chebotayev, and B. Ya. Dubetsky, Appl. Phys. 11, 201 (1976).

${ }^{5}$ V. P. Chebotayev, A. V. Shishayev, B. Ya. Yurshin, and L. S. Vasilenki, Appl. Phys. 15, 43 (1978).

${ }^{6} \mathrm{~J}$. Helmcke, S. A. Lee, and J. L. Hall, J. Opt. Soc. Am. 68, 1635 (1978).

${ }^{7}$ V. P. Chebotayev and B. Ya. Dubetsky, Appl. Phys. 18, 217 (1979).

${ }^{8}$ R. Teets, J. N. Eckstein, and T. W. Hansch, Phys. Rev. Lett. 38, 760 (1977); T. W. Hansch, in Laser
Spectroscopy III, edited by J. L. Hall and J. L. Carlsten (Springer, Berlin, 1977), Vol. 7, p. 149; J. N. Eckstein, A. I. Ferguson, and T. W. Hansch, Phys. Rev. Lett. 40, 847 (1978); M. M. Salour and C. CohenTannoudji, ibid. 38, 757 (1977); M. M. Salour, Appl. Phys. 15, 119 (1978); Rev. Mod. Phys. 50, 667 (1978) ${ }^{9}$ C. J. Borde, C. R. Acad. Sci. 284B, 101 (1977); in Laser Spectroscopy III, edited by J." L. Hall and J. L. Carlsten (Springer, Berlin, 1977), Vol. 7, p. 121.

${ }^{10}$ Ming Chiang Li, Phys. Rev. A 16, 2480 (1977).

${ }^{11} \mathrm{~A}$. Gold, in Quantum Optics, proceedings of the International School of Physics, Enrico Fermi (Course XLII), edited by R. J. Glauber (Academic, New York, 1969).

${ }^{12} \mathrm{~K}$. Shimoda, in High-Resolution Laser Spectroscopy, edited by K. Shimoda (Springer, Berlin, 1976), Vol. 13, p. 11.

${ }^{13}$ S. A. Lee, J. Helmoke, and J. L. Hall, in Laser Spectroscopy $I V$, edited by $\mathrm{K}$. W. Rothe and $\mathrm{H}$. Walther (Springer, Heidelberg, 1979). 